\section{EFEITO DE DIFERENTES MÉTODOS DE RECUPERAÇÃO SOBRE A REMOÇÃO DE LACTATO E DESEMPENHO ANAERÓBIO DE FUTEBOLISTAS}

\author{
EFFECT OF DIFERENTS METHODS OF RECOVERY ON THE LACTATE REMOVAL \\ AND ANAEROBIC PERFORMANCE IN SOCCER PLAYERS \\ EFECTO DE DIVERSOS MÉTODOS DE RECUPERACIÓN SOBRE LA REMOCIÓN \\ DE LACTATO Y EL DESEMPEÑO ANAERÓBICO DE FUTBOLISTAS
}

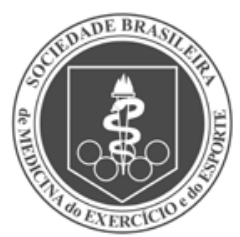

Artigo Original
Homero Gustavo Ferrari

(Educador Físico) $)^{2,3}$

Ramon Oliveira (Educador Físico) ${ }^{1}$

Marcos Vinicius Strapasson

(Educador Físico) ${ }^{1}$

Ricardo Alexandre Rodrigues

Santa Cruz (Educador Físico) $)^{1,4}$

Cleiton Augusto Libardi

(Educador Físico) $^{5}$

Claudia Regina Cavaglieri

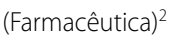

\section{Universidade Metodista de Piracicaba (UNIMEP); Programa de Pós-Graduação em Educação Física - Piracicaba, SP, Brasil. 2. Universidade Estadual de Campinas (Unicamp); Faculdade de Educação Física - Campinas, SP, Brasil. \\ 3. Faculdades Integradas Einstein de Limeira - Limeira, SP, Brasil. \\ 4. Universidade Estadual de Roraima (UERR) - Boa Vista, RR, Brasil. 5. Universidade Federal de São Carlos (UFSCAR) - Departamento de Educação Física - São Carlos, SP, Brasil.}

\section{Correspondência:}

Rua Pedro Guarino, 120

VI. Limerânea, Limeira - SP

CEP: 13482-458.

hgferrari@ig.com.br

\section{RESUMO}

Introdução: O futebol é caracterizado como uma modalidade com intensidades elevadas, realizado de forma intermitente e com sequências aleatórias de fases de esforço e recuperação, dessa forma estratégias que possam de alguma forma minimizar a fadiga muscular, pode fornecer uma importante vantagem competitiva. Objetivo: O presente estudo teve como objetivo comparar diferentes tipos de métodos de recuperação sobre remoção de lactato (La`) sanguíneo e desempenho anaeróbio de futebolistas. Métodos: A amostra foi composta por 23 atletas de futebol entre 16 e 17 anos do sexo masculino, divididos em três grupos: recuperação ativa (RA), passiva (RP) e com gelo (RG). Para a avaliação da capacidade aeróbia foi utilizada a velocidade crítica e para a indução da hiperlactacidemia e desempenho anaeróbio foi utilizando o teste de RAST, aplicado antes (T1) e após (T2) os intervalos de recuperação. As dosagens de La' sanguíneo ocorreram nos minutos dois, quatro, seis, oito e 10 durante a recuperação. A análise de variância ANOVA one-way foi utilizada para a comparação entre as variáveis de caracterização dos grupos. A ANOVA two-way foi realizada para a comparação entre os valores pico e mínimo de La`sanguíneo e o desempenho anaeróbio no T1 e T2 para as três condições de recuperação. Resultados: Não foi verificada diferença significante entre T1 eT2 para a potência máxima e média em nenhum dos grupos estudados ( $p$ > 0,05). Foi verificada maior remoção do La` sanguíneo para a RA (47,62\%), quando comparada a RG (16,9\%; $p=0,001)$ e a RP $(18,20 \% ; p=0,02)$. Conclusão: Pode-se concluir que a RA, RP e RG são eficazes para manutenção do desempenho anaeróbio de jogadores de futebol sub-17. A recuperação ativa se demonstrou mais eficiente para a remoção do La' sanguíneo quando comparada a RP e RG.

Palavras-chave: crioterapia, fadiga muscular, futebol.

\section{ABSTRACT}

Introduction: Soccer is characterized as a sport of high intensity, performed intermittently and with random sequences of periods of effort and recovery; thus, strategies that can somehow minimize muscle fatigue can provide an important competitive advantage. Objective: This study aimed to compare different methods of recovery with the removal of blood lactate ( $\left(\mathrm{C}^{-}\right)$and anaerobic performance of soccer players. Methods: Twenty-three male soccer players between 16 and 17 years were divided into three groups: active recovery (RA), passive recovery (RP) and ice recovery (RG). To assess the aerobic capacity the critical speed was used, and for the induction of hyperlactacidemia and anaerobic performance the RAST test was applied before (T1) and after (T2) the recovery intervals. The measurements of blood La were made at 2, 4, 6, 8 and 10 minutes during the recovery period. The one-way ANOVA was used to compare the variables that characterize the groups. A two-way ANOVA was performed for comparison between the peak and minimum values of blood $L^{\circ}$ and anaerobic performance at $T 1$ and $T 2$ for the three recovery conditions. Results: There was no significant difference between $T 1$ and $T 2$ for maximum and average power in the studied groups $(p>0.05)$. It was observed greater removal of blood $L a^{-}(47.62 \%)$ for the $R A$ when compared to $R G(16.9 \% ; p=0.001)$ and $R P(18.20 \% ; p=0.02)$. Conclusion: $R A, R P$ and $R G$ are effective to maintain the anaerobic performance of soccer players under 17. Active recovery was shown more efficient for removing the blood $L a$ - when compared to $R P$ and $R G$.

Keywords: cryotherapy, muscle fatigue, soccer.

\section{RESUMEN}

Introducción: El fútbol se caracteriza como siendo una modalidad con altas intensidades, realizado de modo intermitente y con secuencias aleatorias de fases de esfuerzo y recuperación; de esta manera, las estrategias, que de alguna forma puedan minimizar la fatiga muscular, pueden dar una importante ventaja competitiva. Objetivo: Este estudio tuvo como objetivo comparar tipos diferentes de métodos de recuperación sobre la remoción de lactato (La') sanguíneo y el desempeño anaeróbico de futbolistas. Métodos: La muestra se compuso de 23 atletas de fútbol, entre 16 y 17 años, del sexo masculino, divididos en tres grupos: recuperación activa (RA), pasiva (RP) y con hielo (RH). Para la evaluación de la capacidad anaeróbica se utilizó la velocidad crítica, y para la inducción de la hiperlactacidemia y el desempeño anaeróbico se usó la prueba de RAST, aplicada antes (T1) y después (T2) de los intervalos de recuperación. Las dosificaciones 
de La sanguíneo ocurrieron en los minutos dos, cuatro, seis, ocho y diez durante la recuperación. El análisis de variancia ANOVA one-way fue utilizado para la comparación entre las variables de caracterización de los grupos. La ANOVA two-way se hizo para la comparación entre los valores pico y mínimo de La sanguíneo y el desempeño anaeróbico, en el $T 1$ y T2, para las tres condiciones de recuperación. Resultados: No se verificó diferencia significativa entre T1 y T2 para la potencia máxima y media en cualquiera de los grupos estudiados $(p>0,05)$. Fue verificada más remoción del La sanguíneo para la $R A(47,62 \%)$, al ser comparada a la $R H(16,9 \% ; p=0,001)$ y la $R P(18,20 \% ; p=0,02)$. Conclusión: Se puede concluir que la RA, RPy RH son eficaces para el mantenimiento del desempeño anaeróbico de jugadores de fútbol sub-17. La recuperación activa se mostró más eficiente para la remoción del La sanguíneo en comparación con la RP y la RH.

Palabras clave: crioterapia, fatiga muscular, fútbol.

\section{INTRODUÇÃO}

O futebol é caracterizado como uma modalidade com intensidades elevadas, realizado de forma intermitente e com sequências aleatórias de fases de esforço e recuperação'. Jogadores profissionais se deslocam em campo em média 11 quilômetros por partida, sendo que desse total 26\% são em alta intensidade, sobretudo, em atividade de aceleração do corpo, o que pode representar em torno de $42 \%$ do total de energia despendida, evidenciando, portanto, uma relevante participação do sistema anaeróbio para o fornecimento de energia ${ }^{2}$. Bangsbo et al. ${ }^{3}$ verificaram em atletas profissionais que as ações de curta duração e alta intensidade podem se repetir de 150 a 250 vezes por partida, evidenciando a alta contribuição dos sistemas da creatina fosfato (CP) e glicólise anaeróbia para a ressíntese de ATP.

A contribuição do sistema anaeróbio durante o jogo também é demonstrada pelas concentrações de lactato (La) sanguíneo. Segundo alguns estudos, essas concentrações podem variar em média de cinco a sete milimolares (mM) por litro 4,5 . O aumento dos níveis de lactato (La־) e prótons de hidrogênio $\left(\mathrm{H}^{+}\right)$produzidos no músculo pode estar relacionado à queda do rendimento do atleta. Essa relação é baseada no fato de que os $\mathrm{H}^{+}$produzidos no músculo poderiam reduzir o $\mathrm{pH}$, tendo como consequência prejuízo na contração muscular ${ }^{6}$. Dessa forma, com a dinâmica do futebol moderno, é inevitável a fadiga muscular durante o jogo, com um aumento mais acentuado no segundo tempo ${ }^{5}$.

Assim estratégias que possam de alguma forma minimizar a fadiga muscular, pode fornecer uma importante vantagem competitiva, uma vez que no futebol um jogo é vencido por meio de uma única ação motora. Dessa maneira, muitos recursos têm sido utilizados no âmbito esportivo com a finalidade de otimizar a recuperação muscular como, por exemplo, recuperação ativa (exercícios de baixa a moderada intensidade), contraste térmico (alternância de exposição ao frio e calor), massagem e crioterapia (aplicação de frio); e, embora a relação do La` e fadiga ainda não estar bem estabelecida, a maioria desses recursos tem sido utilizada com o objetivo de acelerar a remoção do La` sanguíneo ${ }^{6}$.

Dessa forma, o presente estudo teve como objetivo comparar o efeito de três diferentes métodos de recuperação (gelo, passiva e ativa) na remoção do lactato sanguíneo e desempenho anaeróbio de jogadores de futebol da categoria sub-17.

\section{MÉTODOS}

\section{Amostra}

A amostra foi composta por 23 atletas de futebol da categoria sub-17 (idade 16,35 \pm 0,65 anos, estatura de 176,43 \pm 6,03 cm e massa corporal 70,59 $\pm 7,73 \mathrm{~kg}$ ), pertencentes a uma equipe de futebol do interior do estado de São Paulo, participante de competições de nível estadual. A rotina do clube incluía cinco dias de treinamento semanal, com uma média de quatro horas diárias, em duas sessões, além de jogos realizados aos finais de semana. Foram excluídos os goleiros por apresentarem capacidade aeróbia diferenciada em função das demandas exigidas pela posição em campo, e os atletas que apresentaram lesões ou qualquer condição especial de saúde que comprometesse o desempenho nos testes. Todos assinaram o Termo de Consentimento Livre e Esclarecido. Os procedimentos aqui adotados estão de acordo com a Resolução 196/96 do Conselho Nacional de Saúde. O projeto foi aprovado pelo Comitê de Ética em Pesquisa em Seres Humanos sob protocolo 10-05/107.

\section{Desenho experimental}

Todos os atletas foram submetidos ao teste de RAST (T1) para indução da lactacidemia. Em seguida, foram aleatorizados em três grupos para a realização dos diferentes métodos de recuperação com tempo fixo de 10 minutos para todos os grupos.

Recuperação passiva (RP, $n=8)$ : os atletas ficaram em repouso na posição sentada;

Recuperação ativa (RA, $n=8$ ): foi realizada uma corrida com intensidade referente a $80 \%$ da velocidade crítica (Vcrit) em campo de futebol de grama natural em percurso de 20 metros em dinâmica de vai e vem. Durante a RA, a corrida foi interrompida por no máximo 15 segundos para as coletas de sangue; e

Recuperação com imersão em gelo ( $R G, n=7)$ : os atletas ficaram em repouso na posição em pé dentro de um recipiente com água gelada suficiente para cobrir os membros inferiores, com temperatura variando entre $8^{\circ} \mathrm{C}$ e $10^{\circ} \mathrm{C}$. Para o controle da temperatura da água foram realizadas medições a cada dois minutos utilizando um termômetro. Identificado a temperatura abaixo ou acima do estabelecido, era realizada a manutenção da temperatura adicionando gelo ou água quando necessário.

Para os três grupos foram coletadas, durante os intervalos de recuperação, amostras de sangue nos seguintes momentos: imediatamente após e dois, quatro, seis, oito e 10 minutos após o T1 para dosagem de lactato sanguíneo. Em seguida, todos os atletas realizaram novamente o teste de RAST (T2) para verificar o comportamento do desempenho anaeróbio frente aos três protocolos de recuperação. Para a realização da comparação entre os diferentes métodos de recuperação foi utilizado o maior (pico) e o menor (mínimo) valor de lactato sanguíneo de cada sujeito obtido durante a recuperação.

\section{Capacidade aeróbia}

A capacidade aeróbia foi avaliada por meio da velocidade crítica (Vcrit), pelo modelo distância-tempo ${ }^{7}$. Para tanto, os sujeitos foram submetidos a três tiros máximos nas distâncias de 800, 1.600 e 2.400 metros em pista oficial de atletismo de 400 metros. Foram coletados os tempos para cada desempenho para posterior cálculo da Vcrit através da equação matemática de inclinação de reta.

\section{Indução da lactacidemia e desempenho anaeróbio}

Para tanto, foi utilizado o teste de RAST (Running-based Anaerobic Sprint Test), composto de seis tiros máximos de 35 metros com intervalos de 10 segundos entre um tiro e outro, sendo que por meio da massa corporal do avaliado e dos tempos de cada tiro é possível 
calcular a potência pico, média e mínima do avaliado, além do índice de fadiga ${ }^{8,9}$. Para a medição do tempo de cada tiro bem como dos intervalos foram utilizados cronômetros manuais. $O$ teste foi realizado em campo de futebol de grama natural e todos os avaliados utilizaram chuteiras. Para comparação entre os desempenhos, o teste foi aplicado antes e após os intervalos de recuperação.

\section{Coleta de sangue para dosagem de lactato sanguíneo}

Foram coletados $25 \mu$ de sangue do lóbulo da orelha, utilizando-se capilares de vidro heparinizados e calibrados. Após cada coleta, o sangue foi imediatamente armazenado em microtubos do tipo Eppendorff de 1,5 $\mathrm{ml}$, contendo $50 \mu \mathrm{l}$ de solução de Naf 1\%. Em seguida, os tubos foram armazenados em recipiente térmico contendo gelo e levados para o laboratório para a determinação das concentrações de lactato sanguíneo. O lactato sanguíneo foi determinado por meio do analisador eletroquímico modelo YSL 1500 STAT (Yellow Springs, OH, EUA).

\section{Análise estatística}

Inicialmente foi realizado o teste de Shapiro-Wilk para testar a normalidade dos dados. A análise de variância ANOVA one-way foi utilizada para a comparação entre as variáveis de caracterização dos grupos. A ANOVA two-way foi realizada para a comparação entre os valores pico e mínimo de lactato sanguíneo e o desempenho anaeróbio no T1 e T2 para as três condições de recuperação (gelo, passiva e ativa). Sempre que um valor significante de $F$ foi obtido, o post-hoc de Scheffé foi aplicado. Em todas as análises foi adotado nível de significância de $p \leq 0,05$. Para todas as análises foi utilizado o pacote estatístico Statistica 7.0 (Statistica, Tulsa, EUA).

\section{RESULTADOS}

Em relação às variáveis de caracterização dos grupos não foram verificadas diferenças significantes ( $p>0,05)$, demonstrando a homogeneidade dos sujeitos (tabela 1).

Na tabela 2 estão expressos valores de potência máxima e média obtidos no T1 e T2 para os RG, RP e RA. Não foram identificadas diferenças significantes nos valores de potência máxima e média para nenhum dos grupos estudados $(p>0,05)$. A figura 1 apresenta a remoção do lactato sanguíneo após os diferentes métodos de recuperação. Os métodos RG, $\mathrm{RP}$ e RA demonstraram redução do lactato sanguíneo após o período de recuperação ( $p=0,0003, p=0,0004, p=0,0001$, respectivamente). Na comparação entre os métodos de recuperação, não houve diferença significante nos valores pico de lactato sanguíneo ( $p>0,05)$ após o T1. Porém, foi verificada maior remoção do lactato sanguíneo (mínimo) para a RA (47,62\%), quando comparada a RG $(16,9 \% ; p=0,001)$ e a RP $(18,20 \% ; p=0,02)$.

Tabela 1. Valores das variáveis de caracterização dos grupos.

\begin{tabular}{c|c|c|c}
\hline Variáveis & RG & RP & RA \\
\hline Idade (anos) & $16,6 \pm 0,5$ & $16,4 \pm 0,7$ & $16,1 \pm 0,6$ \\
\hline Massa corporal (kg) & $73,1 \pm 9,0$ & $70,6 \pm 9,0$ & $68,4 \pm 4,2$ \\
\hline Estatura (cm) & $180,1 \pm 6,2$ & $175,6 \pm 6,0$ & $174,0 \pm 4,7$ \\
\hline Vcrit $(\mathbf{k m} / \mathbf{h})$ & $13,7 \pm 1,5$ & $12,9 \pm 1,3$ & $12,9 \pm 0,2$ \\
\hline RG, recuperação com gelo: RP ras
\end{tabular}

Tabela 2. Valores de potência máxima (Pmáx) e média (Pméd) antes (T1) e após (T2) a aplicação da recuperação com gelo (RG), passiva (RP) e ativa (RA).

\begin{tabular}{c|c|c|c|c|c|c}
\hline \multirow{2}{*}{ Potência } & \multicolumn{2}{|c|}{ RG } & \multicolumn{2}{c|}{ RP } & \multicolumn{2}{c}{ RA } \\
\cline { 2 - 7 } & T1 & T2 & T1 & T2 & T1 & T2 \\
\hline \multirow{2}{*}{ Pmáx (W) } & 520,1 & $\begin{array}{c}512,8 \\
\pm 73,8\end{array}$ & $\begin{array}{c}496,2 \\
\pm 69,0\end{array}$ & $\begin{array}{c}465,1 \\
\pm 51,7\end{array}$ & $\begin{array}{c}512,5 \\
\pm 55,7\end{array}$ & $\begin{array}{c}482,6 \\
\pm 48,4\end{array}$ \\
\hline \multirow{2}{*}{ Pméd (W) } & $\begin{array}{c}494,6 \\
\pm 65,4\end{array}$ & $\begin{array}{c}478,3 \\
\pm 67,2\end{array}$ & $\begin{array}{c}464,6 \\
\pm 45,7\end{array}$ & $\begin{array}{c}464,9 \\
\pm 47,6\end{array}$ & $\begin{array}{c}465,5 \\
\pm 33,7\end{array}$ & $\begin{array}{c}451,7 \\
\pm 40,1\end{array}$ \\
\hline
\end{tabular}

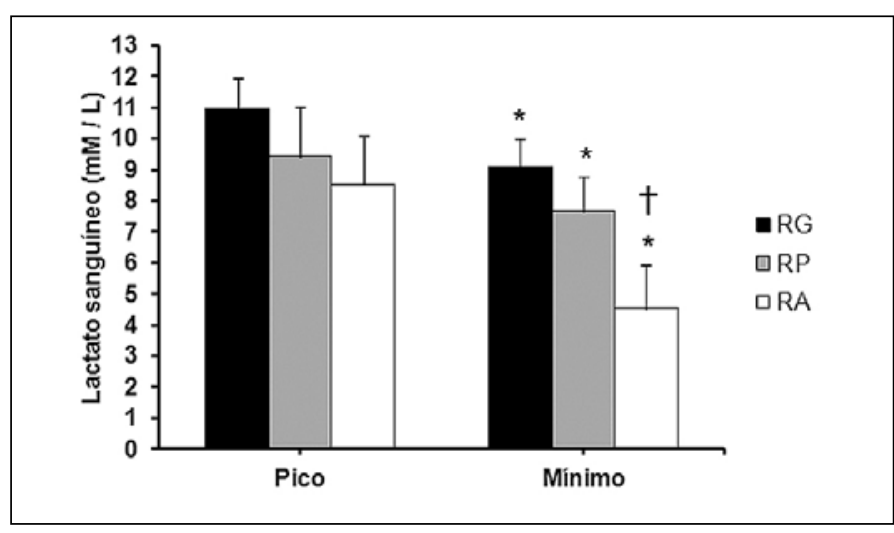

Figura 1. Remoção do lactato sanguíneo após a utilização dos métodos de recuperação com gelo (RG), passiva (RP) e ativa (RA). *Diferença significante comparada ao pico de lactato após o teste RAST. †Diferença significante entre os métodos de remoção. valores em média e desvio padrão.

\section{DISCUSSÃO}

A proposta do presente estudo foi investigar os efeitos da recuperação com gelo (RG), passiva (RP) e passiva (RP) na remoção do lactato (La') sanguíneo e desempenho anaeróbio de jogadores de futebol da categoria sub-17. Os resultados principais encontrados no presente estudo foram: 1) os métodos de recuperação não influenciaram o desempenho anaeróbio de jogadores de futebol da categoria sub-17; 2) a RA demonstrou-se mais eficaz para a remoção do lactato sanguíneo.

Em exercícios intermitentes, a queda no desempenho anaeróbio pode estar relacionada a fatores como microlesões na estrutura contrátil do músculo esquelético, conhecida como dano muscular ${ }^{10}$ e ao acumulo de $\mathrm{H}+$, favorecendo, portanto, a queda do $\mathrm{pH}$ intracelular ${ }^{11}$. Além disso, outros fatores como a depleção de substratos (principalmente glicogênio muscular), hipertermia e acúmulo de vários metabólitos, entre eles: magnésio (Mg2+), adenosina difosfato (ADP), fosfato inorgânico (Pi), amônia $(\mathrm{NH} 3)$ e espécies reativas de oxigênio $(\mathrm{ROS})^{11}$.

Estratégias de recuperação com o objetivo da manutenção do desempenho têm sido amplamente estudadas ${ }^{12-15}$. Em especial a RA e RG (i.e. crioterapia) parecem trazer importantes benefícios para a manutenção do desempenho anaeróbio. Pesquisadores demonstraram que a RG pós-exercício pode atenuar o dano muscular induzido por uma resposta inflamatória devido a uma diminuição da permeabilidade dos vasos sanguíneos e linfáticos ${ }^{16}$. Além disso, tem sido sugerido que a $R G$ pode influenciar o processo regenerativo, que inclui a produção de proteínas de choque térmico e proliferação de células satélites, que são essenciais para o processo de reparação-adaptação ${ }^{17}$. Já a RA parece ser mais efetiva para a remoção do La` sanguíneo em função da manutenção do fluxo sanguíneo acima do repouso, facilitando a captação do La' sanguíneo pelos diversos tecidos para ser oxidado, como, por exemplo, músculo esquelético, músculo cardíaco, cérebro, pâncreas, rins, pulmão, intestino entre outros ${ }^{18}$. No presente estudo, embora a RG e a RP tenham demonstrado menor remoção do La sanguíneo quando comparadas à RA, isso não refletiu na queda da potência máxima e média no T2. Contrariamente aos nossos achados, alguns estudos verificaram queda de desempenho quando a RG é utilizada como método de recuperação. Richendollar et al. ${ }^{13}$ avaliaram os efeitos de quatro diferentes condições de recuperação (gelo, contraste, repouso passivo) no desempenho do salto vertical, agilidade (shutle run) e velocidade (40 jardas). Os resultados apontaram queda no desempenho dos testes neuromotores para ambos os grupos que envolviam a aplicação de gelo. Por outro lado, mais recentemente, foi demonstrado que a RG é considerada um importante método para recuperação dos níveis de força e potência após a realização dos exercícios anaeróbios intermitentes ${ }^{15}$, embora pareça não ser tão efetiva para a remoção do $\mathrm{La}^{-19}$. Resultados similares também foram encontrados por 
Vaile et al. ${ }^{12}$, que verificaram melhor manutenção no desempenho utilizando a $R G$ quando comparado à RA, mesmo com vantagem para a RA na remoção do lactato.

De fato, a queda do desempenho de velocidade após a partida de futebol não foi correlacionada com as alterações de La`e pH muscular; entretanto, foi relacionado com a diminuição dos níveis de glicogênio muscular ${ }^{5}$. Dessa forma, estratégias de intervenção que tenham como objetivo preservar ou acelerar a ressíntese de estoques de glicogênio durante o exercício e entre intervalos de recuperação são importantes para desempenho esportivo tanto em exercícios contínuos como intermitentes ${ }^{5}$. Estudos têm mostrado que a ressíntese de glicogênio muscular é acelerada quando se utiliza intervalos de recuperação passiva em relação à ativa após exercícios de alta intensidade ${ }^{16}$. No presente estudo, não houve melhora no T2 para o GP. O mesmo foi verificado para o grupo $R G$, que embora também tenham ficado em repouso passivo não melhoraram o desempenho no T2. Corroborando com esses achados, Baroni et al. ${ }^{19}$ compararam os efeitos da RG e RP sobre a remoção do La` sanguíneo após exercício de alta intensidade em jogadores de futebol das categorias sub-15 e sub-17. Os resultados mostraram que a RP apresentou decréscimo significativo da concentração de La' sanguíneo, enquanto que o mesmo não foi verificado com a RG. Um efeito bastante evidente da aplicação do frio local na musculatura é a vasoconstrição, e que provavelmente é o efeito que melhor explique a ineficiência da RG na velocidade de remoção do lactato sanguíneo em função da diminuição do fluxo sanguíneo ${ }^{20}$. Entretanto, no presente estudo não foi verificada diferença na remoção do La` sanguíneo entre RG e RP. Embora o tempo de imersão em gelo tenha sido similar (i.e. 10 minutos), é possível que a diferença entre os estudos esteja relacionada à temperatura com que a $R G$ foi realizada.

A maior taxa de remoção foi observada no grupo RA (figura 1). Esse resultado vem ao encontro com a literatura, em que vários estudos têm demonstrado que a recuperação ativa é mais efetiva para a remoção do La` sanguíneo quando comparada à recuperação passiva 14,21,22 em função da manutenção do fluxo sanguíneo acima do repouso, facilitando a captação do La sanguíneo pelos diversos tecidos para ser oxidado ${ }^{18}$. O aumento do fluxo sanguíneo durante a recuperação ativa é maior em relação à recuperação passiva. Além disso, o transporte de lactato é significativamente mais rápido nas fibras musculares oxidativas em relação às fibras glicolíticas. As fibras musculares de contração lenta priorizam a oxidação do lactato, enquanto as fibras musculares glicolíticas primariamente convertem lactato para glicogênio ${ }^{18}$. Considerando que há predomínio da ativação das fibras de contração lenta durante a intensidade de recuperação ativa adotada no presente estudo, pode-se concluir que esse fator foi otimizado nessa intervenção.

A intensidade do exercício em que a recuperação ativa é realizada tem grande influência na velocidade da remoção do La` sanguíneo. Recomenda-se intensidades de 30 a 70\% do consumo máximo de oxigênio $\left(\mathrm{VO}_{2 \text { máx }}\right)^{21}$ e de 80 a 100\% do limiar anaeróbio (Lan) ${ }^{23}$. O teste utilizado no presente estudo para identificação do limiar anaeróbio foi indireto (Vcrit). Estima-se que este superestime em 2 e 9\%24 a máxima fase estável de lactato sanguíneo (MFEL), que é considerada padrão ouro $^{24}$. Entretanto, intensidade de $80 \%$ da Vcrit adotada neste estudo parece ter atingido a recomendação da literatura em relação à faixa de intensidade mais adequada para a recuperação ativa ${ }^{23}$, mesmo que este tenha superestimado a MFEL.

Mesmo a recuperação ativa tendo se demonstrado mais eficaz na remoção do La", para todos os métodos de recuperação investigados (i.e. gelo, passiva e ativa) a potência máxima e média foi mantida noT2. É possível que somente uma sessão teste RAST não tenha promovido fadiga ou dano muscular suficiente para que isso ocorresse, o que pode ter sido um fator limitante do presente estudo. Futuros estudos utilizando uma maior quantidade de estímulos anaeróbios podem ser realizados para verificar se os métodos de recuperação influenciam o desempenho anaeróbio da mesma forma que o presente estudo.

\section{CONCLUSÃO}

Pode-se concluir que a recuperação ativa, passiva e com gelo, são eficazes para manutenção do desempenho anaeróbio de jogadores de futebol sub-17, quando duas sessões são realizadas com curto tempo de recuperação (i.e. 10 minutos). Além disso, a recuperação ativa se demonstrou mais eficiente para a remoção do lactato sanguíneo quando comparada à recuperação passiva e ativa.

Todos os autores declararam não haver qualquer potencial conflito de interesses referente a este artigo.

\section{REFERÊNCIAS}

1. Bangsbo J. The physiology of soccer. Acta Physio Scand 1994;suppl. 151.

2. Osgnach C, Poser S, Bernardini R, Rinaldo R, Di Prampero PE. Energy cost and metabolic Power in elite soccer: a new match analysis approach. Med Sci Sports Exerc 2010;42:170-8.

3. Bangsbo J, Krustrup P, Gonzalez-Alonso J, Saltin B. ATP production and efficiency of human skeletal muscle during intense exercise: effect of previous exercise. Am J Physiol Endocrinol Metab 2001;280:956-64

4. Krustrup P, Zebis M, Jensen JM, Mohr M. Game-induced fatigue patterns in elite female soccer. J Strength Cond Res 2010;24:437-41.

5. Krustrup P, Mohr M, Steensberg A, Bencke J, Kjaer M, Bangsbo J. Muscle and blood metabolites during a soccer game: implications for sprint performance. Med Sci Sports Exerc 2006;38:1165-74.

6. Pastre CM, Bastos FN, Junior JN, Vanderlei LCM, Hoshi RA. Métodos de recuperação pós exercício: uma revisão sistemática. Rev Bras Med Esporte 2009;15:138-144.

7. Kranenburg K, Smith D. Comparison of critical speed determined from track running and treadmill tests in elite runners. Med Sci Sports Exrec 1996;28:614-8.

8. Zacharogiannis E, Paradisis G, Tziortzis S. An evaluation of tests of anaerobic power and capacity. Med Sci Sports Exerc 2004;36:S116.

9. Zagatto AM, Beck WR, Gobatto CA. Validity of the running anaerobic sprint test for assessing anaerobic power and predicting short-distance performances. J Strength Cond Res 2009;23:1820-7.

10. Clarkson PM, Hubal MJ. Exercise-induced muscle damage in humans. Am J Phys Med Rehabil 2002;81:S52-69.

11. Lamb GD. Point: Counterpoint: Lactic acid accumulation is an advantage/disadvantage during muscle activity. J Appl Physiol 2006;100:1410-2.

12. Vaile J, Halson S, Gill N, Dawson B. Effect of cold water immersion on repeat cycling performance and thermoregulation in the heat. J Sports Sci 2008;26:431-40.

13. Richendollar ML, Darby LA, Brown TM. Ice bag application, active warm-up, and 3 measures of maximal

functional performance. J Athl Train 2006;41:364-670.

14. Choi D, Cole KJ, Goodpaster BH, Fink WJ, Costill DL. Effect of passive and active recovery on the resynthesis of muscle glycogen. Med Sci Sports Exerc 1994;26:992-6.

15. Pournot H, Bieuzen F, Duffield R, Lepretre PM, Cozzolino C, Hausswirth C. Short term effects of various wate immersions on recovery from exhaustive intermittent exercise. Eur J Appl Physiol 2011;11:1287-95.

16. Eston R, Peters D. Effects of cold water immersion on the symptoms of exercise-induced muscle damage. J Sports Sci 1999;17:231-8.

17. Toumi H, Best TM. The inflammatory response: friend or enemy for muscle injury? Br J Sports Med 2003;37:284-6.

18. Halestrap AP, Meredith D. The SLC16 gene family - from monocarboxylate transporters (MCTs) to aromatic amino acid transporters and beyond. Eur J Physiol 2004;447:619-28.

19. Baroni BM, Junior ECPL, Generosi RA, Groselli RA, Censi S, Bertolla F. Efeito da crioterapia de imersão sobre a remoção do lactato sanguíneo após exercício. Rev Bras Cineantropom Desempenho Hum 2010;12:179-85.

20. Knight KL. Cryotherapy in Sport Injury Management. Champaign: Human Kinetics, 1995.

21. Baldari C, Videira M, Madeira F, Sergio J, Guidetti L. Blood lactate removal during recovery at various intensities below the individual anaerobic thresshold in triathletes. I Sports Med Phys Fitness 2005;45:460-6.

22. Tessitore A, Meeusen R, Pagano R, Benvenuti C, Tiberi M, Capranica L. Effectiveness of active versus passive recovery strategies after futsal games. J Strength Cond Res 2008;22:1402-12.

23. Menzies P, Menzies C, McIntyre L, Paterson P, Wilson J, Kemi OJ. Blood lactate clearance during active recovery after an intense running bout depends on the intensity of the active recovery. J Sports Sci 2010;28:975-82.

24. Denadai BS, Gomide EBG, Greco CC. The relationship between onset of blood lactate accumulation critical velocity, and maximal lactate steady state in soccer players. J Strength Cond Res 2005;19:364-8. 